\title{
EVALUACIÓN AMBIENTAL DE LA PRÁCTICA "EMBOLSADO" EN PLÁTANO (Musa AAB SIMMONDS). QUINDÍO, COLOMBIA.
}

\author{
ALEXANDER TORRES RODRÍGUEZ $Z^{1}$, MARÍA ELENA \\ BERNAL VERA ${ }^{2}$, ELMER CASTAÑO RAMÍREZ ${ }^{3}$
}

Recibido el 18 de septiembre de 2012 y aprobado el 29 de marzo de 2013

\section{RESUMEN}

En el cultivo de plátano de explotación comercial se utilizan bolsas de polietileno de baja densidad (PEBD) impregnadas con clorpirifos (insecticida) a 1\%, como principal práctica de protección de frutos del ataque de plagas que demeritan su apariencia. No existen estudios que aporten datos sobre la magnitud de los efectos ambientales que causa la técnica de embolsado de plátano en el Quindío, razón por la cual se realizó este trabajo descriptivo analítico mediante un sondeo a partir de encuestas en cinco municipios plataneros de este departamento y seguimiento de la ruta tóxica de las bolsas, donde se cualifica el impacto ambiental generado por este procedimiento, se describe la disposición final de las bolsas tratadas y sin tratar, y se valora la cantidad de plástico que es utilizado en la labor de embolse de racimo en el departamento del Quindío.

El embolsado no presenta mayor utilidad económica para el productor en comparación con la no ejecución de la práctica. Los agricultores en el Quindío que realizan la comercialización a través de intermediarios, no tienen clara la función de la bolsa de polietileno tratada con clorpirifos y sin tratar. El embolse no es una actividad indispensable en el manejo agronómico del cultivo para control de plagas no propagadas por todo el territorio, es una actividad de "moda" generalizada en la zona platanera del Quindío, con impacto ambiental de 840 toneladas/anuales de plástico que entran al sistema más 7,14 toneladas/año de clorpirifos.

\section{PALABRAS CLAVE}

Ruta tóxica, clorpirifos, impacto polietileno.

\section{ENVIRONMENTAL ASSESSMENT OF THE "BAGGING" PRACTICE IN PLANTAIN (MUSa AAB SIMMONDS). QUINDÍO, COLOMBIA.}

\section{ABSTRACT}

Low density polyethylene (LDPE) bags impregnated with chlorpyrifos (insecticide) at $1 \%$ are used in the cultivation of commercial exploitation plantain as the main crops protection practice against pests that detract their appearance. There are no studies that provide data on the magnitude of the 
environmental effects that the plantain bagging technique causes in the Department of Quindío, reason why this descriptive analytical work was conducted with surveys from interviews in five plantain producer municipalities in this region and with environmental monitoring of the toxic route of bags, in which the environmental impact generated with this procedure, is qualified, the final disposal of the treated and untreated bags is described, and the amount of plastic used in the bagging practice in Quindío.is valued.

Bagging does not represent greater economic benefit to the producer compared to the non-implementation of the practice. Quindío producers who carry out commercialization through intermediaries, do not have clarity about the function of the polyethylene bag treated with chlorpyrifos and no treated bags. Bagging is a non-essential activity in the agronomic management to control crop pests which are not spread throughout the region; it is a "fashion" activity widespread in plantain cultivations in the Quindío area, with environmental impact of 840 tons/year of polyethylene that enter in the system more than 7.14 tons/year of chlorpyrifos.

\section{KEY WORDS}

Toxic route, chlorpyrifos, polyethylene impact.

\section{INTRODUCCIÓN}

El polietileno es uno de los materiales más usados por la humanidad. Debido a su carácter polifacético para la fabricación de variados artículos de consumo para el hogar y la agricultura, cuando se desecha se convierte en un componente creciente en los basureros, donde representa $40 \%$ de los residuos sólidos (Zang, Fa \& Wang, 2006).

Desde el punto de vista técnico, la mejor opción para minimizar la contaminación ambiental originada por las bolsas de polietileno, es el reciclaje; para ello existen variados métodos químicos y físico-químicos o de recuperación de energía. Entre los tipos de procesos de reciclado, el más extendido es el reciclaje mecánico, en el que el plástico se recupera a partir de un flujo de residuos y es convertido en copos, gránulos o polvo. Posteriormente se someten a clasificación, trituración y lavado para su transformación en nuevos productos. Este tipo de reciclaje es la mejor opción desde la perspectiva ambiental si se compara con los otros tipos de reciclaje. No obs $\neg$ tante, el reciclado mecánico no está optimizado desde el punto de vista económico (Tukker, 2002); además, los artículos plásticos no pueden ser reciclados indefinidamente, solo se pueden reciclar tantas veces como lo permitan las condiciones físicas y químicas en las que queda el material después de su procesamiento (Segura, Noguez \& Espín, 2007). 
Los residuos o desechos peligrosos (RESPEL) comprenden aquellos sobrantes que por sus características corrosivas, reactivas, explosivas, tóxicas, inflamables, infecciosas o radiactivas pueden causar riesgo para la salud humana y el ambiente. Así mismo, se considera RESPEL a los envases, recipientes y embalajes que hayan estado en contacto con ellos (Ministerio de Ambiente, Vivienda y Desarrollo Territorial, 2007).

Según Castillo, De la Cruz y Clemens (1997), el clorpirifos mantiene sus propiedades después de desechada la bolsa al momento de la cosecha. Se entiende así que las bolsas de polietileno impregnadas con clorpirifos, después de usadas, son RESPEL. Varias investigaciones son concluyentes en afirmar, que el uso continuo de este insecticida organofosforado en la agricultura, genera un alto impacto en el ambiente, la vida animal y la salud humana; desde el año 2000 está siendo sometido a análisis para determinar si continúa en el mercado o le aplican restricciones de uso (Quiñónez, 2005), y a la fecha sigue sujeto a una evaluación general (EPA, 2006). En la zona bananera de Urabá, de acuerdo con un análisis de la actividad de la colinesterasa eritrocitaria realizada a los empleados que manipulan bolsas de polietileno tratadas con clorpirifos, se evidenciaron alteraciones a través del tiempo, razón por la cual las personas dedicadas a la labor del embolse en estas plantaciones, son vulnerables al contacto permanente con estas bolsas (Aguirre \& Narváez, 2009).

\section{Componentes de la bolsa de polietileno}

Está compuesta por petróleo y gas natural (Lajeunesse, 2004) que se calientan en un proceso de rompimiento, que crea monómeros de hidrocarburos (Environmental Literacy Council, 2005). El etileno es el monómero que, después de reaccionar con otras moléculas iguales a él, forma el polímero (polietileno, o simplemente PE). La reacción química para la obtención del PE se llama polimerización. En la estructura de la molécula del $\mathrm{PE}$, la unidad $-\mathrm{CH}_{2}-\mathrm{CH}_{2}$ - se repite indefinidamente y depende del número de moléculas de etileno que reaccionaron entre sí para formar el polímero. El polietileno es el miembro más sencillo del numeroso grupo de resinas termoplásticas (Nordmann, 1971).

El polietileno es un recurso no renovable a partir de etileno (Lajeunesse, 2004) que toma cientos de años en degradarse (Eco-Sense, 2002). Es muy perseguido por fabricantes de elementos para variados usos ya que se puede manipular en cualquier condición, tamaño, forma o color (Greenfeet, 2004). Hay otros dos tipos de polietileno fuera del de alta densidad (PEAD) que es utilizado para hacer bolsas de plástico. El polietileno de baja densidad (PEBD) y el polietileno lineal de baja densidad (PELBD). EI PELBD está construido para bolsas gruesas y brillantes, tales como las de transporte utilizadas por empresas en centros comerciales. EI PEBD, se utiliza para bolsas muy finas o películas que se usan, por ejemplo en bolsas de tintorería (Lajeunesse, 2004). La principal diferencia entre los tres tipos de polietileno (PEAD, PEBD y PELBD) es la 
ramificación de la cadena de moléculas del polímero. La más ramificada de las moléculas es el plástico delgado (Lajeunesse, 2004).

Cuando se termina la vida útil del plástico, es desechado. Estos desechos (PE) no se descomponen en vertederos de basura o en la naturaleza, causando un problema ambiental serio, además ponen en riesgo la vida de 260 animales marinos que podrían comerse los desechos de polietileno o enredar sus cuellos con ellos (Alcántara, 2009). Debido a esto, en algunas ciudades de México ha quedado prohibido el uso de bolsas de polietileno en los supermercados (Grejada, 2009).

\section{Estructura química del clorpirifos}

Fórmula química: $\mathrm{C} 9 \mathrm{H} 11 \mathrm{Cl} 3 \mathrm{NO} \mathrm{PPS}$; nombre químico: (IUPAC): O,O-dietil O-(3,5,6-tricloro-2-piridil) fosforotioato. Sinónimos: O,O-dietil O-(3,5,6-tricloro-2-piridinil) fosforotioato; O,O-dietil O(3,5,6 tricloro-2-piridinil) éster de ácido fosforotioico; Dursban; Brodan; Eradex; Lorsban; Pyrinex. Los productos registrados en Colombia del ingrediente activo clorpirifos para tratar bolsas de polietileno son: Clorpirifos 1\%; Pyritilene 20 blue; Polynsect 1\%® (Ficha Técnica No. CAS: 2921-88-2, 2007).

Evaluación del riesgo ambiental de la bolsa tratada con clorpirifos (Polynsect 1\%® en Ficha Técnica No. CAS: 292188-2, 2007)

La composición de la bolsa plástica es: 1\% de Clorpirifos y 99\% de Polietileno de baja densidad, con las siguientes características de persistencia del clorpirifos de acuerdo con el ambiente en el cual se deposita:

- Suelo: El ingrediente activo clorpirifos tiene un tiempo de vida media de 11 a 141 días, clasificándose como persistente; se reporta una constante de adsorción normalizada Koc entre 995 y $31000 \mathrm{ml} / \mathrm{g}$ indicando que el producto no presenta movilidad en el suelo.

- Agua subterránea: El potencial de lixiviación se calcula con base en la ecuación de Gustafson (GUS), el cual se realiza con base en la Koc entre 995 y $31000 \mathrm{ml} / \mathrm{g}$ y el tiempo de vida media de 141 días; para clorpirifos se obtienen valores de 2,15 y -1,05. El valor de GUS indica que el producto puede presentar un moderado potencial de lixiviación.

- Agua superficial: Clorpirifos tiene un tiempo de vida media mayor que 21 días por fotólisis, lo cual indica que es persistente en ese medio.

- Aire: El valor de la presión de vapor es 2,68 x 10-3 Pa a $25^{\circ} \mathrm{C}$, lo cual indica que clorpirifos puede estar presente en el ambiente en forma de vapor o en forma de partículas.

No obstante, al fabricar el Polynsect $1 \% \AA$, el clorpirifos es atrapado en la estructura de la película plástica, no siendo 
factible su desprendimiento [Resolución No. 0674, MAVDT (2008)].

\section{Antecedentes del clorpirifos}

El clorpirifos es un insecticida organofosforado cuyos residuos han sido asociados con una disminución en los niveles de la Hormona Tiroxina (T4) y con un aumento en los niveles del Estradiol en ovejas. Se ha asociado además con bajo peso al momento del nacimiento y menor circunferencia craneal en niños cuyas madres fueron expuestas en forma crónica al pesticida (Morales \& Rodríguez, 2004). Vindas et al. (2004), encontraron que el clorpirifos induce mayor daño genotóxico en evaluaciones in vitro al compararlo con otros agrotóxicos: imazalil y thiabendazole. Según la Agencia de Sustancias Toxicas y el Registro de Enfermedades (ATSDR, 1997), esta molécula es considerada una sustancia química peligrosa y está sujeta a regulaciones en la ley de agua limpia y la ley federal de los Estados Unidos de control de contaminación de agua. La EPA ha establecido niveles límites de tolerancia para el clorpirifos en las materias primas para la agricultura y los alimentos para seres humanos y animales.

En un estudio realizado a nivel cromosómico de las células somáticas, en trabajadoras de una planta de empaque de banano en Costa Rica expuestas directamente a fungicidas que se aplican a la fruta y además al clorpirifos (insecticida impregnado en las bolsas que protegen los racimos en el campo), reafirman que la exposición ocupacional a los plaguicidas causa daño al material genético, por lo que constituyen un riesgo para la salud (Cuenca \& Ramírez, 2004). Los signos y síntomas que ocasionan estos insecticidas en los seres humanos cuando se trabaja en áreas contaminadas, suelen aparecer cuando el trabajador absorbe el material mediante el contacto repetido con la piel. Al principio los síntomas comprenden cefalea, debilidad y confusión mental, aunque pronto aumentan con más reacciones como: vómito, sudoración profusa, hipersalivación, bradicardia y dolores abdominales tipo cólico (Rosenstein, 1993). Aguirre y Narváez (2009), evidenciaron estos síntomas en investigaciones realizadas a operarios que manipulaban bolsas impregnadas con clorpirifos, en la región bananera de Urabá.

Teniendo en cuenta estos antecedentes, y además al no existir estudios que aporten datos sobre la magnitud de los efectos ambientales que causa la técnica de embolsado de plátano en el departamento del Quindío, se realizó este trabajo donde se cuantificó el impacto ambiental generado por esta práctica con los siguientes logros:

- Se describe la disposición final de las bolsas tratadas y sin tratar usadas en el cultivo del plátano.

- Se valoró la cantidad de plástico que es utilizado en la labor de embolse de racimo en el departamento del Quindío, y se definió la ruta tóxica de las bolsas de 
plátano (lugares de producción, depósito y liberación de contaminantes por el uso del polipropileno).

- Se determinaron los parámetros de compra exigidos por plantas peladoras de plátano en Calarcá (Quindío), que requieren que el racimo de plátano sea embolsado para su posterior procesamiento.

\section{METODOLOGÍA}

\section{Localización de la zona de estudio}

Este trabajo se realizó en los municipios de Armenia, Calarcá, Tebaida, Montenegro y Quimbaya, donde se concentra la mayor parte de la actividad platanera del departamento del Quindío, localizado en el centro geográfico del occidente colombiano. Su economía se basa en la agricultura, donde el cultivo de plátano es el renglón más importante con participación de $57 \%$ sobre toda producción departamental. La zona de estudio se encuentra entre 1000 y 1700 msnm, clima medio-húmedo y con precipitaciones promedio de 2500 mm/año.

\section{Diseño del estudio}

Para determinar el impacto ambiental generado por las bolsas de polietileno en la actividad platanera del Quindío, se ejecutó el siguiente plan:

- Se realizaron treinta (30) encuestas al azar en fincas tipo, a manera de sondeo, seis (6) por cada uno de los municipios con mayor área cultivada de plátano, las que se ubicaron con base en información de la Secretaría de Desarrollo Rural de Armenia; en veredas con características análogas de ecofisiografía y que cultivan el plátano con técnicas similares; los resultados se sometieron a un análisis multivariado.

- La información obtenida a partir de las encuestas arrojó datos sobre: toneladas de plástico por hectárea de plátano situado en el ambiente, cantidad de clorpirifos y metabolitos lanzados al entorno, riesgo de contaminación de operarios y ambiente por deposición de clorpirifos o su metabolito tricloropiridinol (TCP). Estos datos permitieron una proyección sobre el área platanera del departamento del Quindío con la que se determinó el impacto ambiental de la práctica de embolsado de plátano. A la información obtenida se le hizo un análisis de conglomerados por clusters (grados de asociación o similitud).

- Complementariamente se hizo un seguimiento de las bolsas plásticas de desecho (tratadas y sin tratar con clorpirifos), lo que permitió determinar su ruta tóxica con identificación de puntos clave de impacto sobre salud humana, animal y liberación de gases tóxicos. 


\section{RESULTADOS}

Valoración de contaminación regional

\section{Respuestas de los agricultores sobre la labor de embolsado}

De las fincas cultivadas en plátano $40 \%$ pertenecen a pequeños productores con áreas entre 1-5 ha; los medianos (50\%) tienen áreas entre 5-15 ha; $10 \%$ corresponde a grandes con 15-30 ha; según categorías establecidas por Rodríguez y Rodríguez (1999), se asume que la mitad de los fundos encuestados pertenecen a medianos agricultores. Es de resaltar que en la mayoría de las fincas la información fue suministrada por los administradores.

11 fincas (40\%), utilizan bolsas de polietileno tratadas porque ofrecen un mejor control de plagas y contribuyen con la calidad del racimo; mientras que $30 \%$ (9) usan bolsa sin tratar porque cumple el mismo propósito que la tratada y es más barata; $27 \%$ utilizan indistintamente bolsas impregnadas con clorpirifos y sin tratar porque no hay diferencia marginal en el precio del insumo o porque son las que están disponibles en el momento; solo dos productores (3\%) no utilizan bolsas de polietileno porque la unidad productiva es pequeña y no se justifica esta labor. Se infiere que, mayoritariamente, los productores no tienen claridad sobre la razón del uso de bolsas tratadas con insecticida, y obedece más a presiones de mercado por parte de las industrias plásticas que elaboran este insumo, lo que se traduce en una deficiente o nula información en las tiendas agropecuarias sobre el uso y manejo de las variadas presentaciones de las bolsas de polietileno.

La decisión de compra de bolsa tratada y sin tratar con respecto al valor de la misma, no afecta la determinación de compra para $61 \%$ de los productores encuestados; para 25\% de los agricultores sí es un factor de decisión al momento de adquirir el insumo; a 14\% les resulta indiferente. $75 \%$ de los consultados, muestran preferencia por la bolsa tratada, debido a que el valor del insumo no repercute en su decisión; contrario a $25 \%$ que coinciden en la deducción alrededor del costo de la bolsa no tratada, donde el consumo de bolsa tratada está estrechamente relacionado con el valor marginal de la bolsa no tratada y no consideran la finalidad del uso para controlar plagas. De los cultivadores, $96 \%$ indican que una de las varias razones para embolsar los racimos de plátano y con mayor relevancia, es mejorar la calidad; factor asociado al control de plagas y tipo de mercado.

El tiempo que se acorta entre floración y cosecha atribuido al embolse, denominado por los productores como "acelera el racimo", se debe en gran medida a la programación de recolección con indicadores de cosecha (encinte); recogen cada 20 días entre cortes, donde el operario se basa en el color de la 
cinta para realizar la labor, así el racimo no esté en su punto óptimo de cosecha. Cuando no se ejecuta la práctica de embolse ni encintado, los indicadores de cosecha se hacen por método tradicional (visual), relacionado con la pérdida de angularidad de los frutos o el cambio en coloración, cada 20 a 25 días después del último corte. Es importante resaltar que la edad de cosecha oscila entre 14 y 16 semanas después de la floración (Aranzazu et al., 2000), y el desempeño productivo de un clon de plátano en una localidad depende de su genotipo y ambiente, pudiendo variar bajo las diversas condiciones agroclimáticas. El aumento de temperatura por el cambio climático en Colombia (Pérez et al., 1998), ha favorecido este parámetro fisiológico como respuesta a la mayor radiación solar incidente sobre las plantaciones, en zonas donde el ciclo productivo del plátano era más prolongado con respecto al tiempo de cosecha; pero así mismo, el cambio climático ha influido en que hayan épocas atípicas de lluvia con periodos largos de nubosidad, lo que afecta los rendimientos en producción y aumenta el tiempo transcurrido entre floración y cosecha del fruto.

7 productores (23\%), se caracterizan por mostrar una correlación directa entre el tamaño del cultivo y el número de bolsas que consumen, seguramente porque este es el cultivo principal; utilizan entre 100 y 500 bolsas/mes; el rango de bolsas utilizado para el total de productores oscila entre 13.200 y 22.500 bolsas/mes. Entre los cultivadores de plátano en los municipios de Armenia, Calarcá, Montenegro, Quimbaya y Tebaida, $63 \%$ almacenan las bolsas después de usadas en un sito especial en el mismo predio (ramada, rancho, bodega), seguido de $30 \%$ que recicla a través de la Compañía Recipelet en conjunto con la Corporación Autónoma Regional del Quindío (CRQ). Otro 7\% lo regalan a particulares.

De los encuestados, $100 \%$ indican que en toda la labor realizada donde se tiene contacto con bolsas de polietileno tratada con clorpirifos, el operario solo utiliza botas de caucho como única medida de protección para la manipulación de este insumo. 


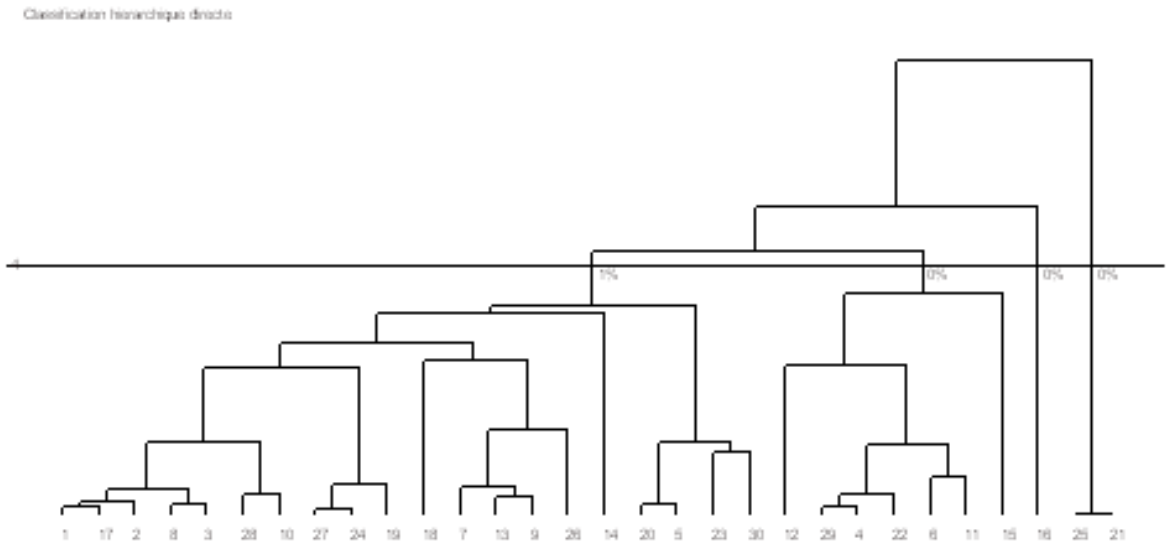

Fuente: esta investigación.

Figura 1. Análisis de clusters.

Mediante un análisis estadístico multivariado, por conglomerados (clusters), se definieron los grados de asociación o similitud de los datos, con los siguientes resultados:

- En general se considera que los datos son homogéneos, ya que la mayoría de predios están agrupados por similitud en área y práctica de embolse, sin embargo hay algunas que se alejan del conglomerado, como es el caso de la finca 21 y la 25 debido a que en las prácticas agronómicas no realizan la labor de embolse.

- Los predios que más se asemejan son las fincas 1 y 17 que utilizan bolsa sin tratar, el valor de la bolsa los hace decidir sobre el uso de la misma; la principal razón de embolsar es el control de plagas y mejorar la calidad; además la finca 17 se caracteriza por el tipo de comercialización.

- Los predios 4 y 29 utiliza bolsas tratadas, el valor no los hace decidir sobre el tipo de bolsa a usar; las principales razones que aducen para embolsar, son la calidad, el control plagas y el tipo de mercado.

\section{Plástico desechado por la labor de embolsado situado en el ambiente}

Mediante promedios con la información obtenida en la encuesta, se determinó que se requiere entre 100 a 500 bolsas/mes para áreas de 1 a 5 ha, dato que se relaciona con el número de veces que se embolsa en el año, lo que al proyectarse sobre el área platanera del Quindío da un estimado de plástico situado en el ambiente de 887 toneladas anuales y 7,14 toneladas/anuales de clorpirifos que corresponde a $85 \%$ de bolsa tratada que se vende en la zona platanera (porcentaje obtenido mediante promedios de cinco tiendas agropecuarias del departamento del Quindío). 
Ruta tóxica, y disposición final que siguen las bolsas de polietileno después de ser utilizadas en la labor del embolse en la zona platanera del Quindío

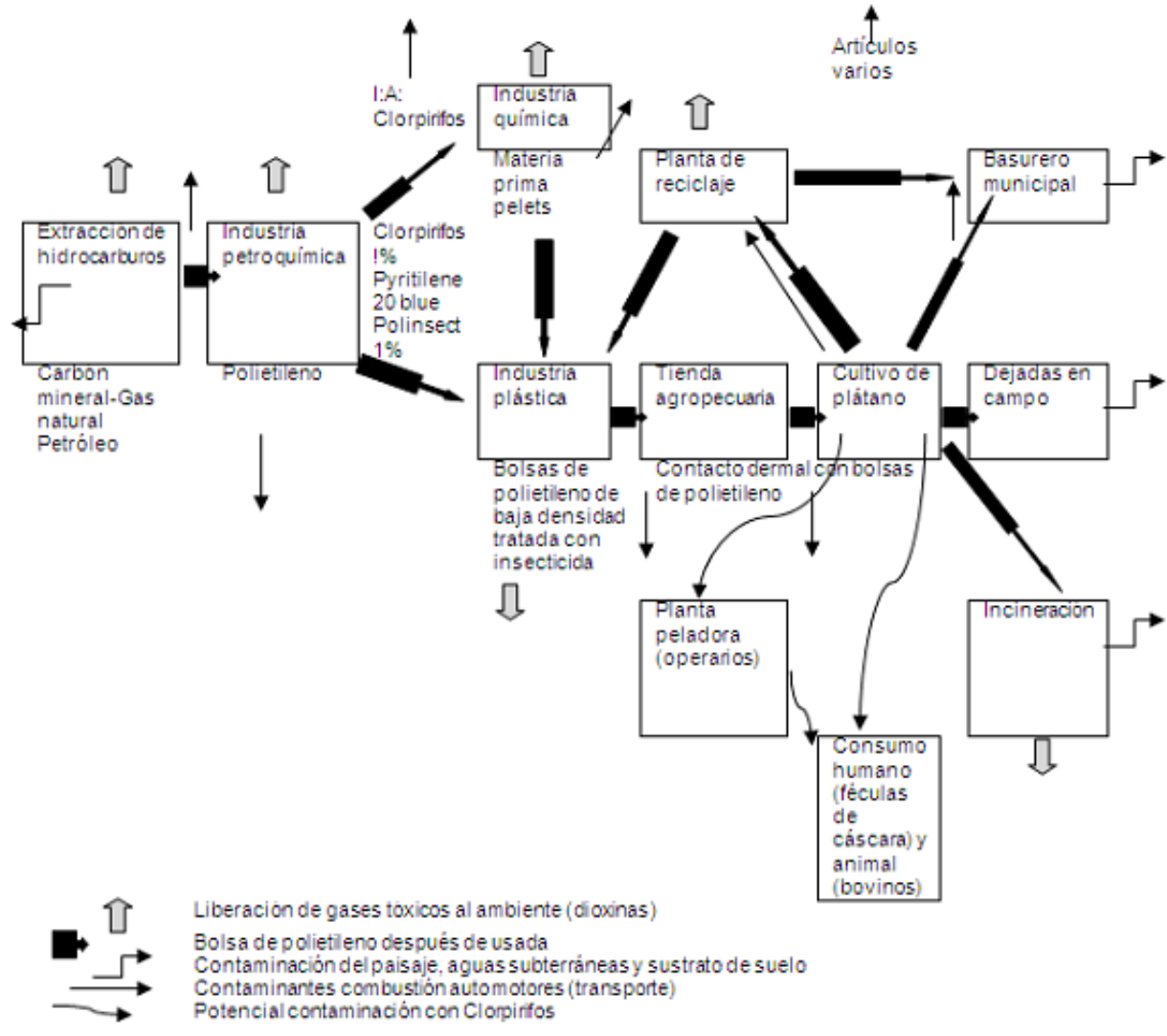

Figura 2. Disposición final bolsas de polietileno.

\section{Artículos elaborados a partir del reciclaje de bolsas de polietileno usadas}

Maquinalsa S.A. es una empresa ubicada en el Quindío, presta un servicio integral en el manejo de residuos sólidos peligrosos en aquellas zonas que presentan problemas ambientales por el uso intensivo de plásticos en el sector rural, logra dar un valor agregado al material reciclado en la fabricación de nuevos productos para ser comercializados en el ámbito nacional e internacional. La planta de reciclaje reprocesa las bolsas de polietileno (tratadas y no tratadas con clorpirifos), recibe contraentrega o retira esta materia prima de las fincas, para la elaboración de material aglutinante, seguido de un proceso de extrusión ${ }^{4}$ para la producción de pelets ${ }^{5}$. El polímero es utilizado en elaboración de baldes para construcción y mangueras para uso agrícola, entre otros ${ }^{6}$. La política para la gestión ambiental de residuos o desechos peligrosos, establece como una de sus estrategias el aprovechamiento y valorización de los residuos peligrosos (RESPEL) con transformación y un tratamiento debido (Ministerio de Medio Ambiente, Vivienda y Desarrollo Territorial, 2005). Sin embargo, algunos trabajos de la 
Universidad Nacional de Costa Rica (Castillo, De la Cruz \& Clemens, 1997) manifiestan que el clorpirifos mantiene sus propiedades después de desechada la bolsa. Aguirre y Narváez (2009), sugieren que el reciclaje de bolsa para la elaboración de otros productos debe garantizar que el material no esté contaminado con clorpirifos, ni por ninguno de los subproductos que genera su descomposición y que pueden afectar usuarios que desconocen el origen del material usado.

\section{Contaminación del suelo-agua-aire}

Las bolsas de polietileno presentan disposiciones variadas en las fincas, con efectos negativos múltiples producto de la incorporación gradual de compuestos nocivos a los sustratos del suelo y aguas subterráneas así: 1) son apiladas en el sitio de recolección del plátano a cielo abierto; 2) otras son dejadas en campo debido a que no se retiran de racimos no aptos para su comercialización por su bajo desarrollo fisiológico; 3) Se dejan en las de plantas volcadas [lo que está asociado a problemas fitosanitarios como nematodos del género Helicotylenchus sp., Pratylenchus sp., Meloidogyne sp., Radopholus sp., reportados en el Quindío, que afectan el anclaje por el daño ocasionado en su sistema radicular según ICA (2000); barrenadores del cormo, entre ellos picudo negro (Cosmopolites sordidus) de importancia económica y se encuentra en $100 \%$ de las áreas productoras de plátano (Alarcón, 2007), donde las galerías formadas en el cormo interrumpen la conexión entre las raíces y el tallo favoreciendo la caída de plantas]; y 4) bolsas que terminan en el suelo en plantaciones con déficit de nutrientes o cultivos ubicados en pendientes, donde las precipitaciones continuas y la gran influencia de vientos hacen que sea de consideración el volcamiento de plantas.

En algunos casos las bolsas se queman en el mismo lugar. Aunque están prohibidas estas prácticas y se someten a sanciones económicas por la Corporación Autónoma Regional del Quindío (CRQ, según Resolución No. 541 de 27 Jun. 2003 Art. $\left.1^{\circ} ; 4^{\circ}\right)$, muchos productores sin conciencia ambiental son indiferentes a estos reglamentos; es de anotar que en muchos casos estas prácticas son realizadas por los empleados de las fincas sin autorización ni conocimiento alguno por parte de los propietarios. De esta forma se liberan al ambiente gases tóxicos, dioxinas y metales pesados, causando efectos que van en detrimento de la salud humana y los ecosistemas.

En algunos casos, las bolsas de polietileno no son retiradas en su totalidad de los racimos en el sitio de producción, y el material plástico llega a la central mayorista de mercado en Armenia (Mercar S.A.) donde es comercializado el producto; el comprador retira las bolsas al desmanar los racimos para su envió en canastillas o bolsas plásticas de $16 \mathrm{~kg}, 18 \mathrm{~kg}, 20 \mathrm{~kg}$, dependiendo del tipo de mercado. A partir de este proceso se generan residuos orgánicos (vástagos), los cuales son mezclados con las bolsas que inicialmente estaban adheridas al 
racimo y son depositados en los contenedores de aseo para su disposición final en los rellenos sanitarios (basurero municipal).

\section{Planta peladora de plátano en Calarcá (Quindío)}

Ryoplat es una empresa agroindustrial ubicada en la zona industrial de Calarcá (Quindío), procesa y comercializa plátano embolsado y sin embolsar adquirido directamente con el productor, quien lo entrega en la planta o esta se encarga del proceso de recolección en las fincas. En el periodo de comercialización de plátano ya sea verde o con principios de madurez que en términos de la cultura del Quindío se nomina "jecho", se deben tener en cuenta algunas variables recomendadas por Ryoplat en la cosecha y postcosecha que afectan la calidad y por consiguiente el precio al venderlo en la planta peladora:

- Falta de experiencia de los trabajadores al cortar el racimo o planta.

- Mal manejo en el transporte del racimo que ocasione lesiones o maltrato del plátano.

- Daños en el fruto por golpe de sol, picaduras por pájaros, roedores, insectos o enfermedades.

Adquirido el producto entra a la planta peladora, para seguir un proceso de selección según la clasificación por tamaño (Peláez et al., 1995) y clasificación por madurez (Tabla 1), para su comercialización al granel en estado verde, maduro y pelado en verde; para este proceso se lava con agua potable, luego se pela y se despunta; una vez pelados se someten a un tratamiento enzimático con ácido cítrico, para evitar el pardeamiento de los frutos; en seguida el producto es enviado a las empresas de fritura ("snacks"). A partir de este proceso se genera un subproducto (cáscara) considerado como producto de desecho agroindustrial (Corpoica, 2002), utilizado para la alimentación animal en bovinos (Figura 3). Es importante resaltar que los plátanos seleccionados para madurar, propios de los frutos climatéricos con característica de mayor aceptación en el mercado, provienen de racimos embolsados por presentar una notoria homogeneidad en el color de la cáscara (amarillo).

Tabla 1. Composición química en la fruta de plátano Dominico Hartón requerido por Ryoplat

\begin{tabular}{|l|c|c|c|}
\hline Composición & Verde & Amarillo & Maduro \\
\hline $\begin{array}{l}\text { Sólidos solubles } \\
\text { totales (\%) }\end{array}$ & 5,0 & 26,0 & 32,0 \\
\hline Azucares totales & 5,0 & 26,0 & 34,0 \\
\hline Almidón (\%) & 68,0 & 62,0 & 57,0 \\
\hline Acidos orgánicos (\%) & 0,6 & 1,2 & 0,8 \\
\hline $\mathrm{pH}$ & 6,2 & 4,6 & 4,5 \\
\hline Hierro, Fe (ppm) & 93,0 & 99,0 & - \\
\hline Calcio, Ca (\%) & 0,21 & 0,14 & - \\
\hline Fósforo, P (\%) & 0,1 & 0,1 & - \\
\hline
\end{tabular}




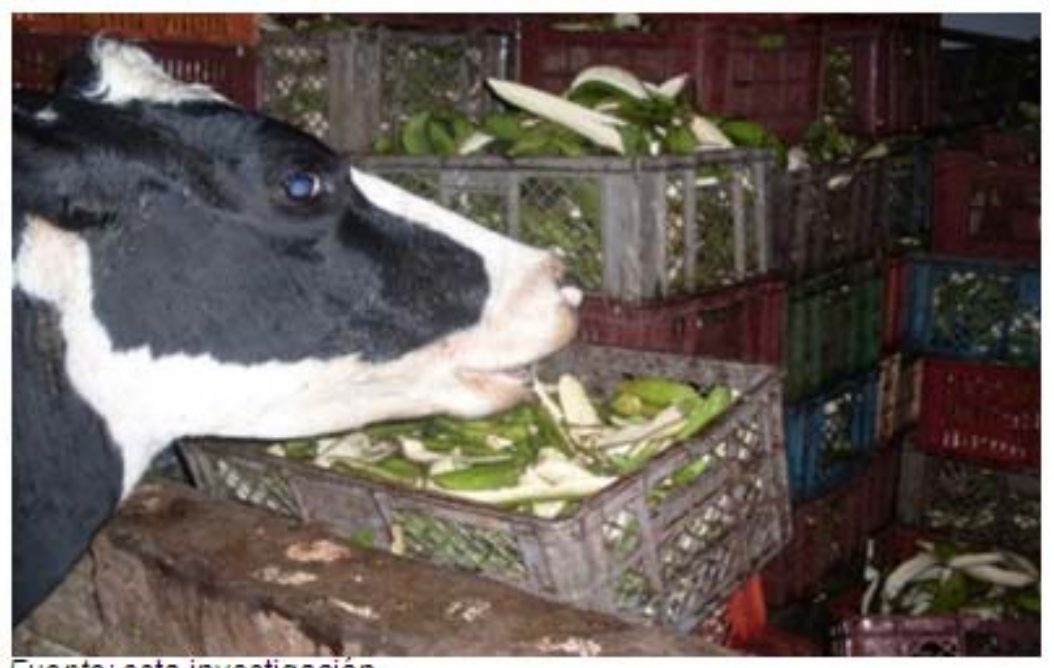

Fuente: esta investigacion.

Figura 3. Desecho agroindustrial (cáscara de plátano).

\section{DISCUSIÓN}

Los productores de plátano tienen un concepto erróneo sobre el uso de la bolsa de polietileno, lo cual se evidencia en la encuesta realizada; no obstante, no hay duda en el consenso de las investigaciones revisadas sobre la mejora de la "apariencia" del fruto, ligado más a un concepto de "estética" que de "calidad". Mientras aquella (la estética) está asociada al embolse, esta (la calidad) se relaciona con desbellote y desmane ejecutados paralelamente con esa práctica. De acuerdo con los resultados obtenidos por Aristizábal (2003), con la técnica de desmane en Dominico Hartón se aumenta la calidad de los frutos e igualmente se infiere que resulta más ventajoso si se tiene en cuenta que, con dicha tarea, se puede prevenir el ataque de enfermedades y plagas que son atraídas por el néctar de las flores (Belalcázar, Valencia \& Lozada, 1991).

Si bien hay una correlación directa entre embolse y el tipo de mercado, también es sabido y reportado, que en el Quindío hay un manejo inadecuado del producto en corte, pésimo acopio en los predios, transporte inapropiado a los centros de distribución, e inexistencia de tecnologías de manejo y conservación. En un seguimiento postcosecha realizado desde Montenegro (Quindío) hasta la bodega mayorista en Cali (Valle), se estableció que en un viaje en vehículo con capacidad de tres toneladas, el racimo recibe en promedio 20 golpes, desde el corte donde se magulla por primera vez, lo que provoca la degradación externa e interna del producto y lo convierte en uno de los aspectos que más incide en la apariencia del plátano con destino al consumo humano en fresco $(\mathrm{CCl}, 2000)$.

En el Quindío no existen reportes de pérdidas económicas ni en producción, asociados al ataque de plagas en frutos de plátano, sin embargo, el control de plagas en frutos representa un rubro 
importante en el costo de sostenimiento del cultivo, inclusive por encima del costo de control de Sigatoka amarilla (Mycosphaerella musicola) y de picudo negro (Cosmopolites sordidus) que son de importancia económica e implican pérdidas significativas en producción. Es debido a la presión del mercado en relación con la "presentación" del producto que se confunde, por parte de los productores, la "calidad" del artículo con la apariencia externa, razón por la cual focalizan prácticas como el embolse de racimos hacia la estética y no hacia el rendimiento.

Según el Ministerio de Medio Ambiente, Vivienda y Desarrollo Territorial (2005), los laboratorios que ofrecen los servicios de análisis físico-químicos de Residuos Peligrosos (RESPEL), en su mayoría no cuentan con soluciones planificadas para la gestión de estos restos. Legalmente, aunque los RESPEL pueden llegar a reciclarse o revalorizarse, su potencial de contaminación permanece activo hasta que esto suceda y deben ser manejados como peligrosos (MAVDT, 2007). La normativa permite a las empresas elaborar diversos artículos a partir de bolsas recicladas de polietileno impregnadas con clorpirifos, entre ellos se fabrican mangueras para uso agrícola las que el agricultor, por su menor costo en el mercado frente a otras mangueras elaboradas de materiales vírgenes, se ve tentado a emplearlas para otros fines como usos domésticos o pecuarios sin la consciente implicación que esto pueda generar en la salud de los seres vivos.

Al extrapolar los consumos de plástico para el departamento del Quindío, se determina que en total ingresan 840 toneladas/anuales de plástico al sistema platanero, más una cantidad de 7,14 toneladas/año de clorpirifos lo que redunda en contaminación visual y ambiental a lo largo de la ruta tóxica donde se incluye contaminación territorial, animal y humana con exposición de operarios y personas por dentro y por fuera de la cadena alimentaria del plátano, sin vigilancia alguna de entidades estatales ni control de los organismos encargados.

A todo esto se agrega que la labor de embolsado solo se cumple como una función estética y no de protección sanitaria, con resultados insustanciales para la agroindustria de pelado y con costos que no se reconocen a través de las mejoras en los precios, convirtiéndose en cargas económicas adicionales para los productores sin compensaciones financieras evidentes, pero sí con sobrecostos para la población, las entidades encargadas de los procesos de recolección de basuras y de las entidades de salud, no auscultadas con este ejercicio. 


\section{CONCLUSIONES}

Los productores de plátano en el Quindío que realizan la comercialización a través de intermediarios, no tienen clara la función de la bolsa de polietileno tratada y sin tratar con clorpirifos.

El embolse de plátano no es actividad indispensable en el manejo agronómico del cultivo, y en el traslado de la bolsa de polipropileno por el departamento del Quindío se deja un paisaje contaminado con plásticos y una larga huella tóxica de clorpirifos a través del paisaje cafetero, con exposición de animales y personas por dentro y por fuera de la cadena alimentaria del plátano, sin vigilancia de entidades estatales ni control de los organismos encargados.

Al sistema platanero entran 840 toneladas/anuales de plástico y 7,14 toneladas/año de clorpirifos lo que deja contaminación por residuos sólidos que el reciclaje de las bolsas de polietileno no soluciona, solo se traslada un riesgo ambiental de la zona rural a zonas urbanas bajo otro grado de transformación.

\section{RECOMENDACIONES}

Se debe divulgar y promover el no uso de bolsa de polietileno en el cultivo de plátano en departamento del Quindío para:

- Proteger el medio ambiente al no generar carga de residuos sólidos y tóxicos en los diferentes ecosistemas (agua-aire-suelo).

- Preservar la salud de operarios (embolsadores) que manipulan bolsas impregnadas con insecticidas (clorpirifos) de potenciales intoxicaciones por ser una sustancia química peligrosa asociada a malformaciones congénitas.

- Evitar sanciones económicas de parte de entidades regionales por el inadecuado manejo de estos residuos sólidos.

- Contribuir a crear conciencia ambiental en los consumidores de plátano, quienes desconocen el impacto ambiental negativo que se genera por el embolsado y en últimas es quien paga el valor adicional de esta práctica para tener una fruta con apariencia "estética" pero con el mismo valor nutricional de un fruto sin embolsar, donde igualmente la "presentación" (cáscara) del producto termina en un basurero o como alimento animal contaminado. El mayor beneficio económico de la práctica de embolse para mejorar la calidad del fruto, se traslada hacia el sector industrial plástico y las plantas de reciclaje que obtienen la materia prima en las fincas lista para su reprocesamiento y sin ningún costo. 


\section{BIBLIOGRAFÍA}

- Aguirre, J. y Narváez, S. (2009). Contaminación en operarios expuestos a insecticidas en el cultivo de banano (Mussa sp.). Caso clorpirifos contenido en la bolsa de campo empleada en la región de Urabá, Antioquia. Tesis, Universidad de Caldas, Facultad de Ciencias Agropecuarias, Programa de Agronomía. p. 40-74.

- Alarcón, J. (2007). Alternativas de manejo integrado de picudo de plátano con énfasis en el control etológico. En: Memorias Seminario Mundial Sobre Plátano y Banano. Montenegro, Quindío. $15 \mathrm{p}$.

- Alcántara, L. (2009). Un mal que dura 1000 años. Obtenido Junio 17, 2011, de http://www.eluniversal.com.mx/notas/577100.html

- Aranzazu, H.; Arcila, P.; Bolaños, B.; Castellanos, C.; Castrillón, A.; Pérez, C.; Rodríguez, M. y Valencia, M. (2000). Manejo integrado del cultivo del plátano. Corporación Colombiana de Investigación Agropecuaria (Corpoica). Regional 9. 78 p.

- Aristizábal, L.M. (2003). Efecto del desmane y la distancia de siembra sobre las características productivas del plátano FHIA-20. Rev Int Bananos y Plátanos INFOMUSA, 13(1), 9-12.

- ATSDR (Agencia de Sustancias Tóxicas y el Registro de Enfermedades). (1997). Departamento de Salud y Servicios Humanos de los EE.UU., Servicio de Salud Pública. Resumen de salud pública, 1997. Clorpirifos caso \# 2921-88-2. Obtenido Junio 7, 2011 de http://www.atsdr.cdc.gov/es

- Belalcázar, C.; Valencia, J. y Lozada, J. (1991). La planta y el fruto. En: Belalcázar, S. (ed.). El cultivo del plátano en el trópico (pp. 45-89). Manual de Asistencia Técnica No. 50. ICA, Armenia, Quindío, INIBAP. Cali: Feriva. Castillo, L.; De la Cruz, E. y Clemens, R. (1997). Ecotoxicoly and pesticides in aquatic ecosystems of Central America. Environmental Toxicology and Chemistry, 16(1), 41-51.

- CCl. (2000). Acuerdo de competitividad de la cadena productiva del plátano en Colombia. Corporación Colombia Internacional (CCl), Ministerio de Agricultura y Desarrollo Rural. Santafé de Bogotá, Colombia. 76 p.

- Corpoica. (2002). Postcosecha, industrialización y uso de subproductos del plátano. Capacitación tecnológica para el mejoramiento del agronegocio del plátano en el Eje Cafetero. Módulo IX. Corpoica Regional Nueve. Obtenido Enero 1, 2012, de http://www.cadenahortofruticola.org/admin/bibli/20a gronegocio_del_platano.pdf

- CRQ. (2003). Resolución No. 541 de 27 de junio de 2003. Art. 10; $4^{\circ}$ "Prohíbe en todo el territorio del departamento del Quindío, la quema, enterramiento y disposición en ríos, quebradas, suelos y vías de la 
bolsa plástica utilizada en el cultivo de plátano banano [...]".

- Cuenca, P. y Ramírez, V. (2004). Aberraciones cromosómicas en trabajadoras expuestas a plaguicidas. Rev. Biol. Trop., 52(3), 623-628. Obtenido Enero 01, 2012 http://www.ots.ac.cr/tropiweb/attachments/volumes/ vol52-3/27-CUENCA-Aberraciones.pdf

- Eco-Sense. (2002). Eco-bag Campaign. Obtenido Abril 20, 2011, de http://ekosvest.com.mk/eko_kesa/eko_kesa_eng.ht m

- Environmental Literacy Council. (2005). Paper or Plastic? Obtenido Abril 20, 2011, de http://www.enviroliteracy.org/article.php/1268.html

- EPA -Environmental Protection Agency, US-. (2006). Insecticidas. 20 oct. 2006. Obtenido Junio 17, 2011, de http://www.epa.gov/oppfod01/safety/spanish/health care/handbook/Spch4.pdf

- Ficha Técnica No. CAS: 2921-88-2. (2007). Obtenido Junio 17, 2011, de http//www2.ine.gob.mx/sistemas/plaguicidas/pdf/clorpirif os etil.pdf

- Greenfeet. (2004). Paper vs. Plastic - The Shopping Bag Debate. Obtenido Junio 17, 2011, http://www.greenfeet.net/newsletter/debate.shtml

- Grejada, E. (2009). Prohíben a comercios dar bolsas de plástico. Obtenido Junio 17, 2011, de http:/lwww.eluniversal.com.mx/ciudad/94564.html

- ICA. (2000). Plagas y enfermedades del plátano. Corporación Colombiana de Investigación Agropecuaria. Boletín de Sanidad Vegetal No. 4. Bogotá. 106 p.

- Lajeunesse, S. (2004). Plastic Bags. Chemical and Engineering News, 82(38), 51.

- Ministerio de Ambiente, Vivienda y Desarrollo Territorial MAVDT-. (2007). Bases conceptuales para la gestión integral de residuos o desechos peligrosos. $183 \mathrm{p}$.

- _ (2008). Resolución No. 0674 de 2008. "Por la cual se emite dictamen técnico ambiental para el producto formulado Polynsect $1 \% \AA$ del ingrediente activo grado técnico clorpirifos, dentro del tramite administrativo de registro". $6 \mathrm{p}$.

- Ministerio de Medio Ambiente, Vivienda y Desarrollo Territorial. (2005). Política ambiental para la gestión integral de residuos o desechos peligrosos. Bogotá, Colombia. $122 \mathrm{p}$.

- Morales, C. y Rodríguez, N. (2004). El Clorpirifos: posible disruptor endocrino en bovinos de leche. Rev Col Cienc Pec, 17(3), 255-266.

- Morton, J. (1999). Procesamiento de plásticos: Inyección, moldeo y PVC. México: Limusa.

- Nordmann, J. (1971). Análisis Cualitativo y Química inorgánica. 6 ed. México: Continental. 422 p.

- Peláez, M.C.; González, G.S.; Díaz, E.I.; Amaya, A. y Giraldo, A. (1995). Comercialización del plátano 
Dominico-Hartón cultivado en el departamento del Quindío. En: Belalcázar, S.; Cayón, G.; Jaramillo, O. y Cortes, C. (eds.). Tecnología del Eje Cafetero para la siembra y explotación rentable del cultivo del plátano (pp. 110-125). CORPOICA, Comité Departamental de Cafeteros del Quindío, CIID (IDRC), INIBAP, INPOFOS. Armenia: Fudegraf.

- Pérez, C.; Poveda, G.; Mesa, O.; Carvajal, L.F. y Ochoa, A. (1998). Evidencias de cambio climático en Colombia: tendencias y cambios de fase y amplitud de los ciclos anual y semianual. Boletín del Instituto Francés de Estudios Andinos, 27(3), 537-546. Obtenido Diciembre 21, 2011, de http:// redalyc.uaemex.mx/redalyc/src/inicio/ArtPdfRed.jsp?iCv $\mathrm{e}=12627318$

- Quiñónez, J. (2005). Efecto del insecticida-acaricida bifentrina y azufre impregnado en la bolsa de polietileno para la protección del racimo de banano (Musa spp.) contra cochinilla harinosa (Pseudococcus sp.) y fumagina (Capnodium sp.). Tesis, Universidad de San Carlos, Facultad de Agronomía, Guatemala. 50 p.

- Rodríguez, A. y Rodríguez, M. (1999). Aspectos socioeconómicos del cultivo del plátano en Colombia. Oficina Regional de Planeación-Corpoica, Regional Nueve. Manizales.

- Rosenstein, E. (1993). Diccionario de especialidades agroquímicas. 4 ed. México: PLM. 679 p.

- Segura, D.; Noguez, R. y Espín, G. (2007). Contaminación ambiental y bacterias productoras de plásticos biodegradables. Biotecnología, 14, 361-371. Obtenido Octubre 15, 2011, de http:// www.ibt.unam.mx/computo/pdfs/libro_25_aniv/capit ulo_31.pdf

- Tukker, A. (2002). Comparing feedstock recycling of plastics waste to mechanical recycling methods. TNO, Holland.

- Vindas, R.; Ortiz, F.; Ramírez, V. y Cuenca, P. (2004). Genotoxicidad de tres plaguicidas utilizados en la actividad bananera de Costa Rica. Revista de Biología Tropical, 52(3), 601-609.

- Zang, L.; Fa, W. y Wang, S. (2006). Novel Photodegradable Low-Density Polyethylene-TiO2 Nanocomposite Film. Environmental Science \& Technology, 40(5), 1681-1685.

1. Ingeniero Agrónomo.

2. Ingeniero Agrónomo M.Sc.

3. Ingeniero Agrónomo Esp. Profesor Titular Universidad de Caldas. elmercr@ucaldas.edu.co

4. Extrusión: Se "fluidiza" (torna en líquido) una resina utilizando un tornillo sinfín dentro de un cilindro largo. Los copos se colocan en la extrusora en el extremo del tornillo con el diámetro más grande y se comprimen mientras se llevan a la boquilla de salida. El calor 
combinado de la fricción producida por el flujo y de las bandas de la calefacción suplementarias provocan la fundición de la resina, extrayéndose de la mezcla los contaminantes volátiles (Morton, 1999).

5. Peletización: El líquido extraído a través de la boquilla tiene las características de un fideo largo. Mientras pasa a través de un orificio, una cuchilla giratoria corta el fideo en segmentos cortos, que caen en un baño de agua donde se enfrían (pelets) (Morton, 1999).

6. Información obtenida vía telefónica por parte de funcionario de la planta de reciclaje Recipelet.

7. Camacho A. (mayo 2012) Información personal Ingeniero de la industria de procesamiento de plátano RYOPLAT 